\title{
Erratum
}

Correction to "The Retention Behavior of Ginsenosides in HPLC and Its Application to Quality Assessment of Radix Ginseng"

The article

Arch Pharm Res Vol 32, No 5, 667-676, 2009

\section{The Retention Behavior of Ginsenosides in HPLC and Its Application to Quality Assessment of Radix Ginseng}

Ping Hu ${ }^{1}$, Guo-An Luo ${ }^{1,3}$, Qing Wang ${ }^{1}$, Zhong-Zhen Zhao ${ }^{2}$, Wan Wang ${ }^{1}$, and Zhi-Hong Jiang ${ }^{2}$

${ }^{1}$ School of Chemistry and Molecular Engineering, East China University of Science and Technology, Shanghai 200237, China, ${ }^{2}$ School of Chinese Medicine, Hong Kong Baptist University, Kowloon Tong, Hong Kong, and ${ }^{3}$ Analysis Center, Tsinghua University, Beijing 100084, China

was incorrectly printed in the issue of APR.

This article must be deleted from the issue.

We regret this error and apologize for any confusion or inconvenience if it may have caused. 